\title{
Behavioral Function of the Components and the Blend of the Sex Pheromone of the Cabbage Looper, Trichoplusia $\mathrm{ni}^{1,2}$
}

\author{
CHARLES E. LINN, JR. ${ }^{3}$, AND LYLE K. GASTON \\ Department of Entomology, University of California, Riverside, California 92521 \\ ABSTRACT \\ Environ. Entomol. 10: 751-755 (1981)
}

Individual male cabbage looper moths, Trichoplusia ni, were tested in a sustainedflight tunnel to the sex pheromone, a 93:7 blend of (Z)-7-dodecenyl acetate and dodecyl acetate. Two experimental procedures were used to investigate the interaction of the two components in the upwind flight response of males. In the first test, chemical components were selectively removed from the moths' flight path at two distances from the source, corresponding to areas of the tunnel in which the blend was or was not active in increasing the number of upwind flights. In the second test, males were offered a choice between plumes, in their normal flight path, which contained different components. Results of both tests showed that males were unresponsive to dodecyl acetate alone at all phases of the flight response, responses to the blend were dependent on the two components being mixed in the same plume, and response to the blend altered the males subsequent flight response to $77-12$ :Ac alone.

A persistent problem in lepidopteran pheromone studies has been the determination of the behavioral role of identified compounds that comprise the pheromone. This problem has grown with the increasing number of species shown to possess multicomponent blends (Roelofs 1979, Cardé 1979). Chemical components have been classified by their degree of influence on the upwind flight behavior exhibited by males, both with respect to long-distance upwind oriented flight or close-range behaviors (Roelofs and Cardé 1977), and early vs. late behaviors (Baker and Carde 1979). Although the association of single components with specific behavior patterns has proved useful, detailed behavioral studies show that compounds do not generally act alone, but that specific blends of compounds function as a unit to evoke specific behaviors, or to enhance the entire sequence of behaviors (Baker et al. 1976, Baker and Cardé 1979, Linn and Gaston 1981, Cardé and Hagaman 1979).

The sex pheromone of the cabbage looper moth, Trichoplusia ni (Hübner), is a 93:7 ratio of $(Z)-7$ dodecenyl acetate (Z7-12:Ac) plus dodecyl acetate (12:Ac) (Bjostad et al. 1980). Behavioral studies using a sustained-flight tunnel suggested that $\mathrm{Z7}$ 12: Ac functioned as a primary component (as defined by Roelofs and Cardé 1977), activating the males and initiating upwind oriented flight. Dodecyl acetate functioned as a secondary component, significantly increasing the number of males that approached closer than $50 \mathrm{~cm}$ to the pheromone source and increasing the time spent in contact with the source when tested in the natural 93:7 blend and optimum dosage (Linn and Gaston 1981). By itself, 12: Ac did not elicit any behavioral response.

In the present study, the behavioral role of the blend was investigated further by more closely ex-

\footnotetext{
2 Lepidoptera: Noctuidae. 22 October 1980

3 Present address: Department of Entomology, N.Y. State Agric. Exp. Str., Geneva, NY 14456.
}

amining the interaction of 12:Ac with $\mathrm{Z7-12:Ac}$ in the upwind flight response of male $T$. ni. Males were found to be unresponsive to 12 :Ac alone at all phases of the flight response; responses to the blend were dependent on the two components being mixed together in the same plume, and perception of the blend significantly changed the males upwind flight response to Z7-12:Ac alone.

\section{Materials and Methods}

The flight tunnel, rearing of moths, testing procedures, and chemicals used here were the same as in the previous study (Linn and Gaston 1981). For all of the present tests a source strength of $10^{-3} \mu \mathrm{g}$ of Z7-12:Ac plus $8 \times 10^{-5} \mu \mathrm{g}$ of 12:Ac (a 93:7 blend) was used, corresponding to the optimum dosage in previous tests.

Two experimental designs were used to test the interaction of the two components. Each procedure was conducted separately, and for each experiment the test situations were presented in a randomized manner during each test period. Results were analyzed within each experiment by using the method of adjusted significance levels for proportions (Ryan 1960).

\section{Removal of Components from the Flight Path}

Two source structures provided the chemical stimulus. One support rod with paired copper discs was placed in a vertical position at the upwind end of the tunnel whereas the other was placed at a right angle to the first with the rod projecting out of a small access door on the side of the tunnel. The disc pairs were positioned $5 \mathrm{~mm}$ apart with the disc surfaces parallel to the airflow, so that material from both sources would mix in a common plume.

Individual moths were allowed to become activated and initiate upwind oriented flight. When the moth had flown within a certain distance of the source ( $35 \mathrm{~cm}$ in some tests, $85 \mathrm{~cm}$ in others), the horizontal rod and discs containing a particular com- 
ponent was pulled rapidly from the flight path and out of the side of the tunnel. The respective distances were selected based on previous work which showed that the greatest effect of the blend on upwind oriented flight occurred within $50 \mathrm{~cm}$ of the source, with close-range flight to the source closer than 25 $\mathrm{cm}$ dependent on the blend (Linn and Gaston 1981).

\section{Choice Tests}

Two source structures were placed vertically at the upwind tunnel position and separated at the midline such that in the regions $85 \mathrm{~cm}$ (sources $12 \mathrm{~cm}$ apart) or $35 \mathrm{~cm}$ (sources $8 \mathrm{~cm}$ apart) downwind, $\mathrm{NH}_{4} \mathrm{Cl}$ smoke plumes from the two sources would merge. Males were exposed individually to various combinations of the two components to determine their ability to discriminate between the contents of two plumes within their normal flight path. Male $T . n i$ typically fly upwind in our tunnel in a zig-zag pattern of oscillations in both the horizontal and vertical directions encompassing a cylindrical $15-\mathrm{cm}$-diameter region of the tunnel extending the length of the flight path. Smoke plumes in our tunnel were generally 5 to $7 \mathrm{~cm}$ diameter at $25 \mathrm{~cm}$ downwind of the source, expanding 2 to $3 \mathrm{~cm}$ at $125 \mathrm{~cm}$ downwind. It was assumed that the smoke plume closely approximated the spatial dimensions of the pheromone plume in the laminar flow conditions of the flight tunnel (Cardé and Hagaman 1979), and that the crosswind flight path exhibited by males was greater than the plume dimensions. This was a desired feature of the design, since one objective was to present the moth with both components within the airspace encompassing its upwind flight path, with the chemicals mixed in one region and relatively separated in another.

\section{Results}

\section{Removal of Components from the Flight Path}

Removal of 77-12:Ac or the blend from the flight path when males were 85 or $35 \mathrm{~cm}$ from the source resulted in termination of all upwind flights ( $n=50$ for each distance). In these tests loss of the chemical signal by removal of the source resulted in a 2 - to 3-sec period of continued upwind flight, followed by sustained stationary flight for 1 to $3 \mathrm{sec}$. Males then began to fly in a crosswind pattern which expanded two to three times in lateral dimension, with the flight path approximating an oval. No net forward progress was observed during this flight pattern. Upwind oriented flight ended with the moth exiting the plume in an upward vertical flight, slightly downwind, to the top of the tunnel.

Removal of a blank source (Fig. 1, test I) when moths were $85 \mathrm{~cm}$ downwind did not appear to affect the upwind flight behavior to Z7-12:Ac or the blend. Significantly more males reached the source with the blend than to Z7-12:Ac alone, as in previous experiments with an equivalent source strength (Linn and Gaston 1981).

Tests with the two components on separate sources are shown in Fig. 1, test II or III. Removal of

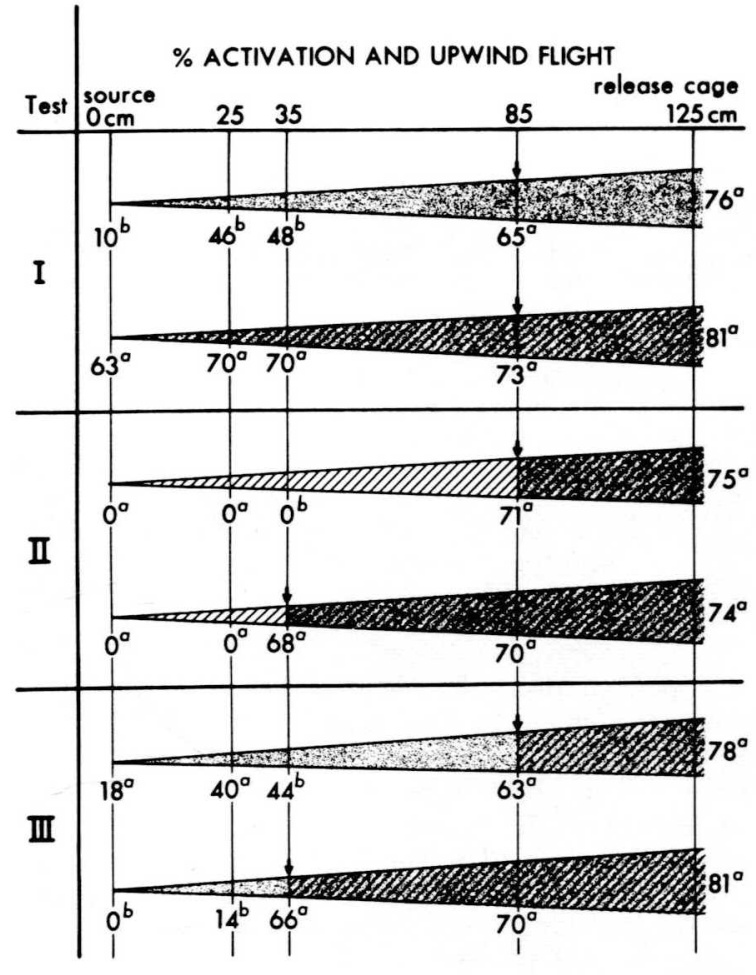

FIG. 1.-Responses of male $T$. $n i$ to sources of pheromone $\left(10^{-3} \mu \mathrm{g}\right.$ of $\mathrm{Z7}-12$ : Ac and $8 \times 10^{-5} \mu \mathrm{g}$ of 12:Ac) after removal of one or both components from the flight path when moths were 85 or $35 \mathrm{~cm}$ downwind of the source (marked by arrows). Time-averaged plumes are drawn to illustrate the presumed boundary (as determined by $\mathrm{NH}_{4} \mathrm{Cl}$ smoke), with the plume contents before (downwind of the arrow) and after (upwind of the arrow) removal of the horizontally placed source $(12: \mathrm{Ac}=$ slashed lines, Z7-12:Ac $=$ shading). For each test $n=50$ for each combination of sources tested. Numbers in a column within a test followed by the same letter are not significantly different at 0.05 level, by the method of adjusted significance levels for proportions.

Z7-12:Ac (test II) from the flight path resulted in termination of upwind flight, at either distance downwind. Moths did not continue to fly upwind to a source of 12:Ac alone. Removing 12:Ac (test III) resulted in different responses at the two distances. With removal at $85 \mathrm{~cm}$ downwind, $40 \%$ of the males flew to within $25 \mathrm{~cm}$ of the source of Z7-12:Ac with $18 \%$ reaching the source, and this was not significantly different from the first test (46 and 10\%). Removing 12:Ac with males $35 \mathrm{~cm}$ downwind (test III), however, resulted in only $14 \%$ of the moths reaching the region near the source of $\mathrm{Z7}-12$ : Ac, with none of the males touching the source. This was significantly fewer than with moths $85 \mathrm{~cm}$ downwind, or the number that would be expected with Z7-12:Ac alone (test I).

The results in Fig. 1 show that male $T$. ni failed to sustain upwind oriented flight to a source of $12: \mathrm{Ac}$ alone after initiating a flight response to Z7-12:Ac or the blend. Further, the blend was required to 
sustain the upwind flight of a significant number of responders after the moths had flown into the tunnel region $50 \mathrm{~cm}$ downwind of the source. For these males, 27-12:Ac alone was no longer sufficient for continued upwind flight to the source.

\section{Choice Tests}

Male $T$. ni made approximately equal numbers of approaches to within $25 \mathrm{~cm}$ of two sources of Z7-12:Ac (21 and $18 \%$ with plume separation at 85 $\mathrm{cm} ; 20$ and $18 \%$ at $35 \mathrm{~cm})$, or to two sources of the blend ( 34 and $28 \%$ at $85 \mathrm{~cm} ; 31$ and $35 \%$ at $35 \mathrm{~cm}$ ). This result was expected since the plume contents from each pair of sources was the same. When responding to two identical sources males appeared to exhibit zig-zag flight in a larger space than that observed with a single source, presumably because material merging from the two sources formed a larger plume (approximately 15 to $17 \mathrm{~cm}$ ) downwind of $50 \mathrm{~cm}$ from the source. Males did not appear to exhibit directed flight to a source of equal strength until they were within $35 \mathrm{~cm}$.

In tests with 27-12:Ac and the blend on separate sources (Fig. 2, test I), significantly more males approached and touched the source releasing the blend, with no significant difference between plumes separated at 85 or $35 \mathrm{~cm}$ downwind. With 12:Ac and the blend on separate sources (Fig. 2, test II), all of the males responded to the blend, with no difference between plumes separated at 85 or $35 \mathrm{~cm}$ downwind.

With Z7-12:Ac and 12:Ac on separate sources (Fig. 2, test III), and plume separation $85 \mathrm{~cm}$ downwind, none of the moths oriented to the 12: Ac source and the percentage of upwind flights and source contacts to Z7-12:Ac was not significantly different from that expected in a test with $\mathrm{Z7}-12$ : Ac alone ( 43 vs. $46 \%$, and 9 vs. $10 \%$ ). The males zig-zag flight pattern appeared to be the same as that exhibited to a single source of Z7-12:Ac. When plume separation occurred $35 \mathrm{~cm}$ downwind, however, only $12 \%$ of the moths continued to fly as close as $25 \mathrm{~cm}$ of the Z7-12:Ac source with none reaching the source, significantly fewer than in the $85 \mathrm{~cm}$ test.

The results of tests in Fig. 2 support those obtained in Fig. 1. None of the males oriented to or approached a source of $12:$ Ac alone, and for a significant number of males, both components mixed together in the same plume, and not simply present in the same region of the flight path, were necessary for sustained flight after the moth had entered the region of the tunnel in which the presence of the blend significantly increased the level of response (based on Linn and Gaston 1981).

\section{Discussion}

In a previous study, 12:Ac alone did not evoke an activation response in male $T$. ni when tested over a wide range of dosages, nor did it affect the level of activation to any Z7-12:Ac treatment (Linn and Gaston 1981). The present study shows, further, that moths were not responsive to 12 :Ac alone at any stage of the flight response. It was at first thought

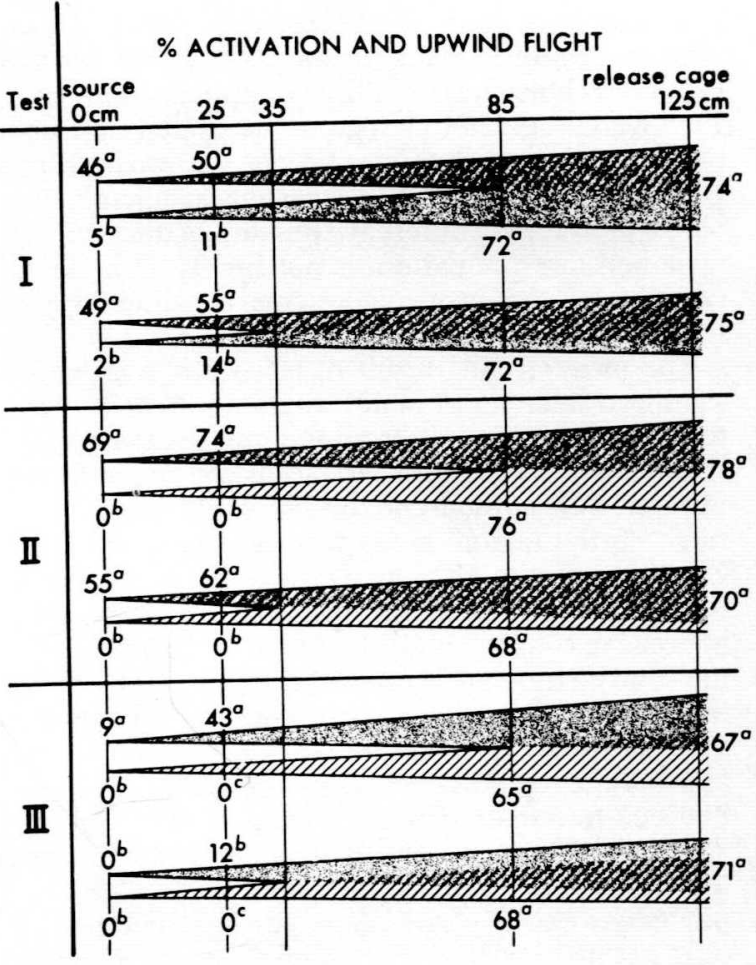

Fig. 2.- Responses of male $T$. $n i$ to choice tests between two sources positioned in the tunnel so that a common plume formed 35 or $85 \mathrm{~cm}$ downwind of the sources. Timeaveraged plumes are drawn to show the presumed boundaries (as determined by $\mathrm{NH}_{4} \mathrm{Cl}$ smoke) and the contents of the two plumes (12:Ac $=$ slashed lines, Z7-12:Ac = shading). For each test $n=65$ for each pair of sources. Numbers in a column, within a test, followed by the same letter are not significantly different at the 0.05 level, by the method of adjusted significance levels for proportions

that 12 :Ac might in part function to replace $\mathrm{Z} 7-12$ :Ac as the predominant stimulus in the region of the flight tunnel close to the source, since 12:Ac was known to evoke an antennal EAG response (Linn and Seabrook, unpublished data) and since the number of upwind flights decreased rapidly close to a source of Z7-12:Ac alone (Linn and Gaston 1981). Data presented in Fig. 1 and 2, however, suggest that the increased percent response with the 93:7 blend compared with that of Z7-12:Ac alone was not due simply to 12 :Ac replacing $\mathrm{Z7-12:Ac}$ as the dominant stimulus. Rather, the blend appears to function as a distinct entity, producing a different stimulus pattern in the central nervous system of the insect.

$(Z)$-7-Dodecenyl acetate is a primary component of the sex pheromone in a number of noctuid species (Roelofs 1979). The data in Fig. 2 suggest that male $T$. ni can discriminate between two closely adjacent plumes containing predominantly the same chemical compound. The effectiveness of the blend in sustaining upwind oriented flight, however, was dependent on the two components being mixed in the 
same plume, a result also reported for another noctuid moth, Spodoptera litura (Nakamura 1979). From the choice tests in Fig. 2, it appears that male $T$. $n i$ were not able to integrate the stimuli from the two components when in adjacent but separate regions of the flight path, suggesting again that the blend produces a different stimulus pattern in the male and that the pattern is not simply an additive function of the two components presented separately.

The most striking result of this study was that a significant number of males would not sustain flight to Z7-12:Ac alone after apparently responding to the blend. Only $12 \%$ of the moths continued flying upwind while normally 40 to $50 \%$ would be expected to reach the region $25 \mathrm{~cm}$ from a source releasing Z7-12:Ac alone. Thus, perception of the blend not only resulted in sustained upwind flight, close-range approach, contact, and attempted copulation with the source (Linn and Gaston 1981), but also altered the males' responsiveness for the duration of the flight in progress, rendering $\mathrm{Z7}-12$ :Ac alone an inappropriate signal. That males were making a choice between terminating or continuing upwind flight to Z7-12:Ac is evidenced in part by the behaviors exhibited during flight termination. Male T. ni exhibited two clearly differentiated flight patterns in terminating upwind flight. In one pattern the male hovered for 1 to $3 \mathrm{sec}$, thereafter flying in a crosswind casting path in ever-widening dimensions, eventually flying upward and out of the plume. This behavior pattern is similar to that described by Kennedy and March (1974) and occurs when the pheromone stimulus is removed from the flight path. In the second pattern the moth hovered for 1 to $3 \mathrm{sec}$ and then flew upward and out of the plume directly, without exhibiting any crosswind casting. This second pattern is characteristic of termination while the moth is flying upwind toward the source or, as in the case of Fig. 2, test III, when the blend was removed and a plume containing Z7-12:Ac remained. It is suggested that the crosswind casting behavior was exhibited by males attempting to relocate a lost (but appropriate) signal, whereas in the second pattern, termination was not due to loss of the signal, but was instead a response to an inappropriate signal.

As defined by Roelofs and Cardé (1977), secondary components are "chemicals emitted by an insect that are not essential for eliciting upwind anemotaxis but that in combination with the primary component(s) evoke other aspects of the mating sequence." The criterion of being in combination with the primary component appears to be essential. In $T, n i$, 12: Ac was only effective in eliciting behaviors close to the source when mixed with Z7-12:Ac and did not appear to contribute to long-range upwind flight (see Linn and Gaston 1981). As a chemical, 12:Ac would satisfy the definition of a secondary component. However, it is suggested from the present study, with respect to the existing behavioral information for $T$. $n i$, that the functional components of the sex pheromone are $\mathrm{Z7-12:Ac}$ for long-range at- traction and the 93:7 blend for short-range behaviors. This conclusion is in agreement with that of Nakamura (1979), who showed that $S$. litura also uses a single primary component (Z9-11 TDDA) for long-range attraction and a 10:1 mix of 29-11 TDDA + Z9-E12 TDDA for close-range orientation and mating. These two-component systems contrast with other well-studied Lepidoptera in which a mixture of isomers is essential for long-range attraction, with an additional short range component, as in the redbanded leafroller, Argyrotaenia velutinana (Baker et al. 1976), or a Z-E mixture plus additional components, all of which are essential for upwind flight, close-range orientation, and hairpencilling, as in the Oriental fruit moth, Grapholitha molesta (Baker and Carde 1979). It is apparent that several strategies are possible for the use of multicomponent sex pheromones in the precopulatory sequence of behaviors exhibited by males.

\section{Acknowledgment}

We thank P. Baumer and C. Stern for their assistance in rearing the insect culture, Thomas $C$. Baker for his support and critical review of this manuscript, and Louis Bjostad for his comments and help during the research. Research supported by the Rockefeller Foundation and National Science Foundation grant BNS 76-10268.

\section{REFERENCES CITED}

Baker, T. C., and R. T. Cardé. 1979. Analysis of pheromone-mediated behaviors in male Grapholitha molesta, the Oriental fruit moth (Lepidoptera: Tortricidae). Environ. Entomol. 8: 956-968.

Baker, T. C., R. T. Cardé, and W. L. Roelofs. 1976. Behavioral responses of male Argyrotaenia velutinana to components of its sex pheromone. J. Chem. Ecol. 2: 333-352.

Bjostad, L., L. K. Gaston, L. L. Noble, J. H. Moyer, and H. H. Shorey. 1980. Dodecyl acetate, a second pheromone component of the cabbage looper moth, Trichoplusia ni. J. Chem. Ecol. 6: 727-734.

Cardé, R. T. 1979. Behavioral responses of moths to female-produced pheromones and the utilization of attractant-baited traps for population monitoring. In $\mathbf{R}$. L. Rabb and G. G. Kennedy [eds.], Movement of highly mobile insects: concepts and methodology in research. Univ, Graphics, North Carolina State University, Chapel Hill. pp. 286-315.

Cardé, R. T., and T. E. Hagaman. 1979. Behavioral responses of the gypsy moth in a wind tunnel to airborne enantiomers of disparlure. Environ. Entomol. 8: $475-484$.

Kennedy, J. S., and D. Marsh. 1974. Pheromone-regulated anemotaxis in flying moths. Science 184: 999-1001.

Linn, C. E. Jr., and L. K. Gaston. 1981. Behavioral re. sponses of male Trichoplusia ni in a sustained-flight tunnel to the two sex pheromone components. Environ. Entomol. (in press).

Nakamura, K. 1979. Effect of the minor component of the sex pheromone on the male orientation to pheromone source in Spodoptera litura (F.). Chem. Rev. Insects (Russia) 4: 153-156. 
Roelofs, W. L. 1979. Pheromones and other chemical attractants: pheromone trap specificity and potency. In R. L. Rabb and G. G. Kennedy [eds.], Movement of highly mobile insects: concepts and methodology in research. Univ. Graphics, North Carolina State University, Chapel Hill. pp. 272-285.
Roelofs, W. L., and R. T. Cardé. 1977. Responses of Lepidoptera to synthetic sex pheromone chemicals and their analogues. Annu. Rev. Entomol. 22: 377-405.

Ryan, J. A. 1960. Significance tests for multiple comparison of proportions, variances and other statistics. Psychol. Bull. 57: 318-328. 